\title{
Parity meter for charge qubits: An efficient quantum entangler
}

\author{
B. Trauzettel, ${ }^{1,2}$ A. N. Jordan, ${ }^{3,4}$ C. W. J. Beenakker, ${ }^{1}$ and M. Büttiker ${ }^{3}$ \\ ${ }^{1}$ Instituut-Lorentz, Universiteit Leiden, P. O. Box 9506, 2300 RA Leiden, The Netherlands \\ ${ }^{2}$ Kavli Institute of Nanoscience, TU Delft, P. O. Box 5046, 2600 GA Delft, The Netherlands \\ ${ }^{3}$ Département de Physique Théorique, Université de Genève, 1211 Genève 4, Switzerland \\ ${ }^{4}$ Institute for Quantum Studies, Texas A\&M University, College Station, Texas 77843-4242, USA \\ (Received 22 February 2006; revised manuscript received 26 April 2006; published 16 June 2006)
}

\begin{abstract}
We propose a realization of a charge parity meter based on two double quantum dots alongside a quantum point contact. Such a device is a specific example of the general class of mesoscopic quadratic quantum measurement detectors previously investigated by Mao et al. [Phys. Rev. Lett. 93, 056803 (2004)]. Our setup accomplishes entangled state preparation by a current measurement alone, and allows the qubits to be effectively decoupled by pinching off the parity meter. Two applications of the parity meter are discussed: the measurement of Bell's inequality in charge qubits and the realization of a controlled-NOT gate.
\end{abstract}

DOI: $10.1103 /$ PhysRevB.73.235331

PACS number(s): 03.65.Ta, 73.23. - b, 03.65.Yz

\section{INTRODUCTION}

Recently, it has been realized that parity meters based on solid state structures should be very promising candidates to create entanglement of electronic systems. ${ }^{1-3}$ Whereas previous proposals and applications of parity meters in the solid state have dealt with the entanglement of the spin degree of freedom, we presently investigate a parity meter based on charge qubits, which is able to generate entanglement in a solid state system just by measuring a dc current through a quantum point contact (QPC). The setup under consideration, schematically shown in Fig. 1(a), is a particular example of the general class of mesoscopic quadratic quantum measurement devices investigated by Mao et al. in Ref. 4. The design of our device has been inspired by the work of Ruskov and Korotkov, where it has been demonstrated that current and noise measurements of a QPC coupled to two charge qubits can be used as an entangler. ${ }^{5}$ We propose a setup in which this task can be achieved by a current measurement only.

The charge qubits, e.g., a single electron in a tunnelcoupled double quantum dot (DQD), are coupled capacitively to the measurement device (the parity meter). Recently, coherent quantum oscillations have been measured in DQD systems. ${ }^{6}$ Quantum detectors based on QPC's coupled to DQD qubit systems have been studied intensively in the past, both theoretically ${ }^{7}$ as well as experimentally. ${ }^{8}$ Transport properties of Coulomb-coupled DQD systems have also been analyzed. ${ }^{9}$

Generally speaking, the idea of a parity meter is that it can distinguish between the subspaces of two parity classes of quantum states but it cannot distinguish between different states in each parity class. It has been demonstrated that such a device can be used to implement a controlled-NOT (CNOT) gate. ${ }^{1} \mathrm{~A}$ CNOT gate is a universal quantum gate, and therefore enables universal quantum computation when combined with single-qubit gates.

The design of the parity meter that we propose in this paper is very straightforward. It just relies on two qubit systems (based on DQD's) and a single measurement device (based on a QPC). All elements of the parity meter can be built with standard lithographic techniques in the laboratory. If we think about these qubits as DQD's in GaAs heterostructures then dephasing due to coupling of the charge on the dots to acoustic phonons ${ }^{10}$ and dephasing due to background charge fluctuations ${ }^{11}$ cause severe problems. Nevertheless, charge qubits based on DQD's in other structures such as carbon nanotubes ${ }^{12}$ or semiconductor nanowires might have much better coherence properties (due to the fact that they are essentially one-dimensional systems), which would make our predictions measurable. Recently, charge qubit operations of an isolated (leadless) silicon double quantum dot have been reported with an extremely long coherence time. ${ }^{13}$

The paper is organized as follows. In Sec. II, we propose a specific realization of a charge parity meter, i.e., a quadratic quantum measurement device. Subsequently, in Sec. III, we demonstrate how the parity meter acts as an entangler. In Sec. IV, we discuss two applications of the parity meter: (i) the measurement of a violation of Bell's inequality and (ii) a realization of a CNOT gate. We conclude in Sec. V.

\section{REALIZATION OF A CHARGE PARITY METER}

It has been pointed out in Ref. 4 that a general quadratic quantum measurement device provides a simple way of entangling two otherwise noninteracting qubits. In such a measurement device, the transmission amplitude $t$ of some par-

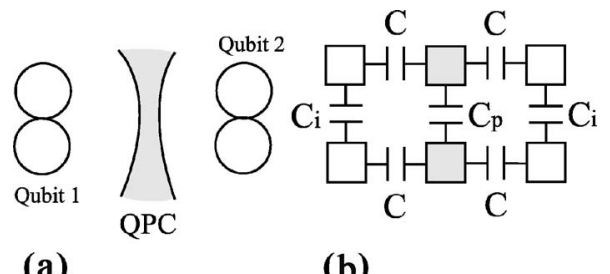

(a)

FIG. 1. Parity meter setup. In (a), two DQD's are shown with a QPC in between them. By measuring the current through the QPC, we are able to determine the parity class of the two-qubit state formed by a single electron in each DQD qubit. In (b), a capacitive model is illustrated for the setup considered in (a). The two gray boxes in the middle correspond to the dipole across the QPC. 
ticles, e.g., electrons, should depend on the measurement basis operators $\sigma_{z}^{(\alpha)}$ of the two qubits $\alpha=1,2$ in the following way:

$$
t \propto \sigma_{z}^{(1)} \sigma_{z}^{(2)} .
$$

In this section, we demonstrate that a physical realization of a parity meter for charge qubits consists of two DQD's and a single QPC. The setup we have in mind consists of two DQD's alongside a QPC; cf. Fig. 1(a). We will show that a capacitance model based only on linear relations between charge and potentials explains the existence of the desired quadratic coupling (1) in our device. While in Ref. 4 the quadratic measurement is achieved by considering an inflection point of the transmission with $d t / d U=0$, where $t$ is the transmission amplitude and $U$ the potential of the measurement device, the quadratic coupling which we discuss below is realized for arbitrary transmission since it is a consequence of the spatial symmetry of our arrangement. A similar idea, using geometric symmetry (in a more complicated interferometric structure) to realize a quadratic detector has been proposed in Ref. 14.

We assume that each DQD contains a single electron, which therefore acts as a charge qubit, and can be described by the Hamiltonian

$$
H_{\mathrm{QD}, \alpha}=-\frac{1}{2}\left(\epsilon_{\alpha} \sigma_{z}^{(\alpha)}+\Delta_{\alpha} \sigma_{x}^{(\alpha)}\right) .
$$

Here $\alpha=1,2$ (for the two different qubits), $\epsilon_{\alpha}$ is the difference of single-particle energy levels in each dot, $\Delta_{\alpha}$ is the tunnel coupling between the dots, and $\sigma_{x}^{(\alpha)}$ is a Pauli matrix acting on qubit $\alpha$. The direct electrostatic coupling between the two DQD's is neglected. However, the two qubits are indirectly coupled to each other via the QPC.

The corresponding coupling term, in our setup, may be written as

$$
H_{\mathrm{int}}=(\Delta \hat{E} / 2) \sigma_{z}^{(1)} \sigma_{z}^{(2)},
$$

where $\Delta \hat{E}$ is a charging energy operator with a quantum expectation value $\Delta E=E_{E}-E_{O} \equiv\langle\Delta \hat{E}\rangle$ equal to the difference in charging energy of the even (index $E$ ) and odd (index $O$ ) parity class. In the tunneling regime, the operator $\Delta \hat{E}$ can be associated with the standard detector input variable $\lambda a_{R}^{\dagger} a_{L}$ + H.c., where $\lambda$ is the coupling constant, $a_{R}^{\dagger}$ is the creation operator for an electron in the one lead of the detector, and $a_{L}$ an annihilation operator in the other lead. ${ }^{24}$ The combined Hamiltonian of the two DQD's, the QPC, and the coupling (3) then reads

$$
H=H_{\mathrm{QD}, 1}+H_{\mathrm{QD}, 2}+H_{\mathrm{QPC}}+H_{\mathrm{int}},
$$

where $H_{\mathrm{QPC}}$ is the Hamiltonian of the detector.

To justify the interaction Hamiltonian (3) in the case of an arbitrary two-qubit parity detector, the coupling Hamiltonian may be expressed in the basis, defined with each of the configurations $\{|\uparrow \uparrow\rangle,|\downarrow \downarrow\rangle,|\uparrow \downarrow\rangle,|\downarrow \uparrow\rangle\}$, where we assign pseudospins to the position of the electron in the DQD: spin $\uparrow$ corresponds to the case where the electron is in the upper dot and spin $\downarrow$ corresponds to the case where the electron is in the lower dot. The even parity class contains the states $\{|\uparrow \uparrow\rangle,|\downarrow \downarrow\rangle\}$, while the odd parity class contains $\{|\uparrow \downarrow\rangle,|\downarrow \uparrow\rangle\}$. We will show below that the parity detector can distinguish only between the even and the odd parity classes. This defines the basis that is accessible to the parity detector. The parity detector cannot distinguish the states in either even or odd subclass, but it can distinguish between parity subclasses. In particular, the charging energy operator of both even configurations is the same, and the charging energy operator of both odd configurations is the same, but the even energy operator is (in general) not equal to the odd energy operator. Therefore the coupling Hamiltonian can be expressed as

$$
H_{\text {int }}=\hat{E}_{E}(|\uparrow \uparrow\rangle\langle\uparrow \uparrow|+| \downarrow \downarrow\rangle\langle\downarrow \downarrow|)+\hat{E}_{O}(|\uparrow \downarrow\rangle\langle\uparrow \downarrow|+| \downarrow \uparrow\rangle\langle\downarrow \uparrow|) .
$$

Introducing the projection operators $P_{\uparrow, \downarrow}^{(\alpha)}$ on the up or down state of qubit $\alpha$, going to sum and difference variables $\hat{\Sigma}$ $=\left(\hat{E}_{E}+\hat{E}_{O}\right) / 2, \Delta \hat{E}=\left(\hat{E}_{E}-\hat{E}_{O}\right)$, and recalling that $P_{\uparrow}^{(\alpha)}+P_{\downarrow}^{(\alpha)}$ $=\mathbf{1}^{(\alpha)}, P_{\uparrow}^{(\alpha)}-P_{\downarrow}^{(\alpha)}=\sigma_{z}^{(\alpha)}$, we find that the coupling Hamiltonian may be rewritten as

$$
H_{\mathrm{int}}=\hat{\Sigma} \mathbf{1}^{(1)} \mathbf{1}^{(2)}+(\Delta \hat{E} / 2) \sigma_{z}^{(1)} \sigma_{z}^{(2)} .
$$

The first term may be absorbed into the detector Hamiltonian, and the second term recovers our coupling Hamiltonian (3).

We will now demonstrate that a single QPC placed in a special way between two DQD's has a Hamiltonian of the form (3). As illustrated in Fig. 1(b), we divide the two DQD's as well as the QPC into two regions with two corresponding charges each. The left DQD has charge $Q_{1}=-Q_{2}$ $=Q_{L}$ in dots with potentials $U_{1}$ and $U_{2}$. The right DQD has charge $Q_{3}=-Q_{4}=Q_{R}$ in dots with potentials $U_{3}$ and $U_{4}$. We set $e \equiv 1$ and $\hbar \equiv 1$. Suppose that, in the odd parity class with $Q_{L} Q_{R}=-1$, the QPC has a potential $V(x)$ along the $x$ axis given by ${ }^{15}$

$$
V(x)=V_{0}-\frac{1}{2} m \omega_{x}^{2} x^{2}+\mathcal{O}\left(x^{4}\right) .
$$

We are interested in the change of the potential $V_{0}$ at the saddle point as the qubit configuration changes from the odd to the even parity class. We call the saddle point potential of the even parity class $V_{1}$. The saddle point potentials allow us to determine the transmission coefficient of the QPC.

As compared to the odd parity class, the even class will polarize the QPC, i.e., there will be an additional electrical field $E$ at the saddle point with a potential $V(x)=V_{0}+e E x$ $-\frac{1}{2} m \omega_{x}^{2} x^{2}+\cdots$, which will give rise to a shift in the location of the saddle point and an increase in its height,

$$
V_{1}=V_{0}+\frac{e^{2} E^{2}}{2 m \omega_{x}^{2}} .
$$

To estimate the field $E$ we consider the capacitive model shown in Fig. 1(b). The QPC dipole is described with a capacitance $C_{p}$ and the dipole charges $Q_{5}=Q_{d}$ and $Q_{6}=-Q_{d}$ exist in regions with potentials $U_{5}$ and $U_{6} .{ }^{16} \mathrm{~A}$ charge on a 
dot next to a dipole region has a capacitance $C$ and the interaction of the charges across a DQD is described by an (internal) capacitance $C_{i}$. For simplicity, we assume that only nearest neighbor charge configurations couple to each other. This is justified because the QPC screens the DQD's, and, therefore, decreases the direct coupling between the DQD's substantially. We now have for the DQD charges the equations

$$
\begin{gathered}
Q_{1}=C_{i}\left(U_{1}-U_{2}\right)+C\left(U_{1}-U_{5}\right)=Q_{L}, \\
Q_{2}=C_{i}\left(U_{2}-U_{1}\right)+C\left(U_{2}-U_{6}\right)=-Q_{L}, \\
Q_{3}=C_{i}\left(U_{3}-U_{4}\right)+C\left(U_{3}-U_{5}\right)=Q_{R}, \\
Q_{4}=C_{i}\left(U_{4}-U_{3}\right)+C\left(U_{4}-U_{6}\right)=-Q_{R},
\end{gathered}
$$

and for the QPC dipole charges

$$
\begin{gathered}
Q_{5}=C_{p}\left(U_{5}-U_{6}\right)+C\left(U_{5}-U_{1}\right)+C\left(U_{5}-U_{3}\right)=Q_{d}, \\
Q_{6}=C_{p}\left(U_{5}-U_{6}\right)+C\left(U_{5}-U_{1}\right)+C\left(U_{5}-U_{3}\right)=-Q_{d} .
\end{gathered}
$$

These become a complete set of equations if we assume that the region over which the dipole extends has a density of states $D$ such that a small variation of the potential in these regions gives rise to a charge $Q_{5}=-D U$ and $Q_{6}=D U$ with $U=\left(U_{5}-U_{6}\right) / 2$. Here we have assumed that the QPC potential is spatially symmetric in the odd parity configuration. This requires that the QPC is located symmetrically in between the DQD's and permits us to take the density of states to the right and the left of the QPC to be equal to $D \equiv D_{L}$ $=D_{R}$.

We obtain for the Coulomb energy

$$
E_{c}=\frac{1}{2} \sum_{i} Q_{i} U_{i}=\frac{Q_{L}\left(Q_{L}+C U\right)+Q_{R}\left(Q_{R}+C U\right)}{\left(2 C_{i}+C\right)}-D U^{2},
$$

where

$$
\begin{gathered}
U \equiv U_{5}=\frac{C}{C_{\text {sum }}^{2}}\left(Q_{L}+Q_{R}\right), \\
C_{\text {sum }}^{2}=\left(2 C_{p}+2 C+e^{2} D\right)\left(2 C_{i}+C\right)-2 C^{2} .
\end{gathered}
$$

We see that there exist a contribution to the Coulomb energy proportional to $Q_{L} Q_{R}$ given by

$$
\Delta E_{c}=\frac{C^{2}}{C_{\mathrm{sum}}^{4}} \frac{\left(2 C_{i}+C\right) C_{p}+2 C_{i} C}{\left(2 C_{i}+C\right)} 4 Q_{L} Q_{R} .
$$

This contribution affects the saddle point potential in such a way that the QPC acts as a parity meter. Equation (14) can be identified with Eq. (3).

We now estimate the saddle point potential, in the even configuration, which determines the transmission. The voltage drop of the dipole is $2 U$ and, assuming that the center of its charges is separated by a distance $2 d$, we find an electric field $E=U / d$. Hence, using Eqs. (8) and (12) we obtain

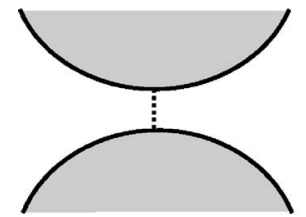

odd case

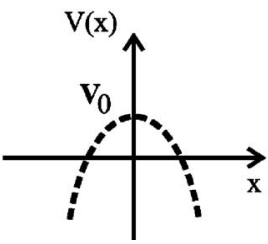

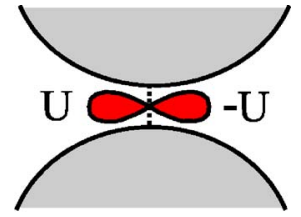

even case

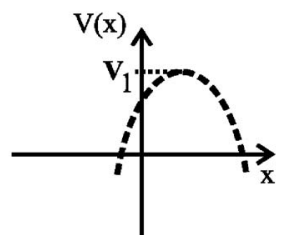

FIG. 2. (Color online) Upper part: Top view of QPC in the parity meter setup. In the odd parity class, where $\left(Q_{L}, Q_{R}\right)$ is $(1$, $-1)$ or $(-1,1)$, no dipole is generated across the QPC and it is nicely symmetric. In the even parity class, where $\left(Q_{L}, Q_{R}\right)$ is $(1,1)$ or $(-1,-1)$, the situation is different. There, a dipole is generated across the QPC by the particular position of the electrons in the quantum dots. The dipole shifts the saddle point to the right (left) depending on the direction of the polarization of the dipole, but for both polarizations the shift is to higher energies. Note that the QPC in this figure is rotated by $90^{\circ}$ with respect to the QPC in Fig. 1. Lower part: Illustration of the saddle point potential for the two configurations.

$$
V_{1}=V_{0}+\frac{1}{2 m \omega_{x} d^{2}}\left(\frac{C}{C_{\text {sum }}^{2}}\right)^{2}\left(Q_{L}+Q_{R}\right)^{2} .
$$

Thus, the QPC has a saddle point height $V_{0}$ for the odd configuration $\left(Q_{L} Q_{R}=-1\right)$ and a somewhat higher saddle point potential $V_{1}$ for the even configuration $\left(Q_{L} Q_{R}=1\right)$. We illustrate in Fig. 2 the symmetric potential landscape of the QPC in the odd case and the generated dipole across the QPC in the even case as well as the corresponding saddle point potentials. The transmission probability $T=|t|^{2}$ of the QPC is directly related to the saddle point height via ${ }^{15}$

$$
T_{\alpha}=\frac{1}{1+e^{-2 \pi\left(E_{F}-V_{\alpha}\right) / \omega_{x}}},
$$

where $E_{F}$ is the Fermi energy in the QPC, $\alpha=0$ for the odd case, and $\alpha=1$ for the even case. This shows that the transmission probability through the system considered here has indeed the desired property stated in Eq. (1).

To summarize, there are three physically distinguishable situations: (i) There is no charge dipole (both odd configurations), (ii) there is a QPC charge dipole pointing up (the even configuration $|\uparrow \uparrow\rangle)$, and (iii) there is a QPC charge dipole pointing down (the even configuration $|\downarrow \downarrow\rangle$ ). Although all three situations are distinguishable in principle, the crucial fact is that the potential height experienced by the transport electrons is the same in both even configurations. Therefore, the current differs only between the even and the odd configuration. For later reference, we define here the two currents

$$
I_{\text {odd }}=\frac{2 e^{2}}{h} V_{\text {bias }} T_{0}
$$




$$
I_{\text {even }}=\frac{2 e^{2}}{h} V_{\text {bias }} T_{1},
$$

where $V_{\text {bias }}$ is the bias voltage across the QPC and we have reintroduced $e$ and $\hbar$ for clarity. During the measurement, the QPC should be operated in the regime of linear $V_{\text {bias }}$ but still $V_{\text {bias }} \gg \Delta$. The reason is that a large bias would break the symmetry between the $|\uparrow \uparrow\rangle$ and the $|\downarrow \downarrow\rangle$ states, so the parity meter could then distinguish the two states of the even class. In typical systems (for instance the ones investigated in Ref. 8), $\Delta \approx 10 \mu \mathrm{eV}, V_{\text {bias }} \approx 1 \mathrm{mV}$, and the $I-V$ characteristic of the QPC is linear. Thus, the above stated requirements can be easily met. The parity measurement time ${ }^{7}$

$$
T_{M}=\frac{4 S_{I}}{\left(I_{\text {odd }}-I_{\text {even }}\right)^{2}},
$$

with $S_{I}=\int d t\langle\Delta I(t) \Delta I(0)\rangle$, and $\Delta I(t)=I(t)-\langle I\rangle$ is the time scale required to obtain a signal-to-noise ratio of order 1 . In current (present day) GaAs-based quantum dot devices this time scale will be of the order of a few microseconds (see, e.g., Ref. 17) and therefore much longer than typical coherence times of the order of a few nanoseconds. For quantum dots in other physical systems such as carbon nanotubes or nanowires the coherence times should be much longer and therefore the measurement time $T_{M}$ can, in principle, become of the same order as the coherence time or even smaller.

For the Bell measurement (discussed below), it is important that the coupling between the two qubits (the two DQD's) can be turned off after the state has been prepared. In our setup, the coupling between the two DQD's is minimized by removing the bias voltage $\left(V_{\text {bias }}=0\right)$ and by pinching off the QPC, therefore, reducing $\Delta E$. For all operations that are done on a time scale $\Delta t \ll 2 / \Delta E$, the density matrix of the two DQD's experiences little coherent evolution due to the coupling term (3). Therefore, on such short time scales the two qubits would behave as if they were decoupled.

\section{PARITY METER AS ENTANGLER}

At the symmetry point of the two-qubit system, where $\epsilon_{1}=\epsilon_{2}=0$ and $\Delta_{1}=\Delta_{2} \equiv \Delta$, our device acts as an entangler just by measuring the current through the QPC.

The Hamiltonian of the two qubits at the symmetry point (index SP) reads

$$
H_{\mathrm{SP}}=-\frac{\Delta}{2}\left(\sigma_{x}^{(1)}+\sigma_{x}^{(2)}\right) .
$$

The eigenstates of $H_{\mathrm{SP}}$ are the two antisymmetric Bell states

$$
\begin{aligned}
& \left|\Psi_{B 1}\right\rangle=\frac{1}{\sqrt{2}}(|\uparrow \downarrow\rangle-|\downarrow \uparrow\rangle), \\
& \left|\Psi_{B 2}\right\rangle=\frac{1}{\sqrt{2}}(|\uparrow \uparrow\rangle-|\downarrow \downarrow\rangle) .
\end{aligned}
$$

The state $\left|\Psi_{B 1}\right\rangle$ belongs to the odd parity class and the state $\left|\Psi_{B 2}\right\rangle$ to the even one. Thus, both eigenstates of the Hamiltonian $H_{\mathrm{SP}}$ can be distinguished from each other by a current measurement through the QPC in Fig. 1. The other two symmetric Bell states

$$
\begin{aligned}
\left|\Psi_{B 3}\right\rangle & =\frac{1}{\sqrt{2}}(|\uparrow \downarrow\rangle+|\downarrow \uparrow\rangle), \\
\left|\Psi_{B 4}\right\rangle & =\frac{1}{\sqrt{2}}(|\uparrow \uparrow\rangle+|\downarrow \downarrow\rangle)
\end{aligned}
$$

are transformed into each other, obeying the time evolution

$$
|\Psi(t)\rangle=\cos (\Delta t+\phi)\left|\Psi_{B 3}\right\rangle-i \sin (\Delta t+\phi)\left|\Psi_{B 4}\right\rangle .
$$

Here, $\phi$ is an arbitrary phase.

Since the states (21)-(24) form a complete basis of the two-qubit system, we can conclude that a measurement of the current through the QPC can take three possible outcomes: (i) $I_{\text {odd }}$, which means that the system is driven into the steady state (21) by the measurement, (ii) $I_{\text {even, which }}$ means that the system is driven into the steady state (22) by the measurement, and (iii) $I_{\text {mix }} \equiv\left(I_{\text {odd }}+I_{\text {even }}\right) / 2$ (with $I_{\text {odd }}$ $<I_{\text {mix }}<I_{\text {even }}$ ), which means that the system is driven into the state (25), which exhibits a dynamical detector signal that is both oscillatory and noisy.

This behavior can be demonstrated within a simple model of parity measurement state preparation with a series of projective parity measurements. Using a master equation description of the time evolution of the density matrix of the two-qubit system, we have verified that the latter model is in agreement with continuous weak measurement. In the master equation description, we have treated the electrons in the QPC as bath variables and integrated out the bath degrees of freedom in the weak coupling and Markovian regime. The resulting master equation is of the Lindblad form, where the decay rates are proportional to the autocorrelation function of the input variable of the parity detector. The algorithm of our model of parity measurement state preparation is as follows.

(1) Fix the desired initial state in the measurement basis. It can be randomly chosen, or can be fixed as a state that is experimentally simple to prepare.

(2) Apply a unitary transformation to change to the Bell basis, where Hamiltonian evolution is simple. More explicitly, if we represent an arbitrary state as

$$
|\Psi\rangle=a|\downarrow \downarrow\rangle+b|\downarrow \uparrow\rangle+c|\uparrow \downarrow\rangle+d|\uparrow \uparrow\rangle
$$

a simple basis transformation enables us to write the same state as

$$
|\Psi\rangle=\alpha\left|\Psi_{B 1}\right\rangle+\beta\left|\Psi_{B 2}\right\rangle+\gamma\left|\Psi_{B 3}\right\rangle+\delta\left|\Psi_{B 4}\right\rangle .
$$

Then, the time evolution in the Bell basis is simply given by

$$
\begin{aligned}
|\Psi(t)\rangle= & \alpha\left|\Psi_{B 1}\right\rangle+\beta\left|\Psi_{B 2}\right\rangle+\gamma\left[\cos (\Delta t)\left|\Psi_{B 3}\right\rangle-i \sin (\Delta t)\left|\Psi_{B 4}\right\rangle\right] \\
& +\delta\left[\cos (\Delta t)\left|\Psi_{B 4}\right\rangle-i \sin (\Delta t)\left|\Psi_{B 3}\right\rangle\right] .
\end{aligned}
$$

(3) Apply Hamiltonian evolution with a randomly chosen time.

(4) Transform back to the measurement basis.

(5) Do a parity measurement: Find the probability of getting the result even $(E)$ or odd $(O)$ from the state; use these 
probabilities to choose a random outcome $E$ or $O$; and then based on the result, update the state.

(6) Transform back to the Bell basis.

(7) Repeat the algorithm from step 2.

As mentioned before, this algorithm gives three possible outcomes: (i) the parity meter measures $O$ all the time $\rightarrow$ state $\left|\Psi_{\mathrm{B} 1}\right\rangle$ has been prepared, (ii) the parity meter measures $E$ all the time $\rightarrow$ state $\left|\Psi_{\mathrm{B} 2}\right\rangle$ has been prepared, and (iii) the parity meter measures a string of a mixture of $O$ and $E$ results $\rightarrow$ a dynamical superposition of the states $\left|\Psi_{\mathrm{B} 3}\right\rangle$ and $\left|\Psi_{\mathrm{B} 4}\right\rangle$ has been prepared, which is not a steady state of $H_{\mathrm{SP}}$. A long sequence of either $O$ 's or $E$ 's, corresponding to cases (i) or (ii), indicates a statistically confident preparation of a Bell state.

A statistical analysis of our model shows that if we start with a product state in one of the two parity classes, e.g., $|\uparrow \downarrow\rangle$ in the odd class, which can be easily prepared experimentally, then the parity meter drives the system with probability $1 / 2$ into the Bell state $\left|\Psi_{\mathrm{B} 1}\right\rangle$. (The same holds for the other parity class and the Bell state $\left|\Psi_{\mathrm{B} 2}\right\rangle$.)

If we, however, start with a random state, e.g., a fully mixed state, then the parity meter still accomplishes a Bell state preparation of the two states $\left|\Psi_{\mathrm{B} 1}\right\rangle$ and $\left|\Psi_{\mathrm{B} 2}\right\rangle$ with a success probability of $1 / 4$ each. If there are nonideal symmetries, e.g., $\Delta_{1} \neq \Delta_{2}$, then, on longer time scales, there will be random switching between the different parity classes.

Before proceeding to the next section, we briefly note that the needed symmetry in the coupling constants between detector and each of the qubits may be tested by dc current measurements, using gate voltages to force the quantum dots into each of the four classical configurations. If there is any asymmetry, this will show up in a slight current difference when comparing the different configurations. The difference of the couplings constants can by slightly tuned with the use of top gates.

\section{APPLICATIONS OF THE PARITY METER}

We describe two applications of the parity meter of interest for quantum information processing. The first one is a proposal to test Bell's inequality in the solid state. The second one is an example of a realization of a CNOT gate using QPC's and DQD's as building blocks.

\section{A. Testing Bell's inequality}

A slight modification of our setup as schematically shown in Fig. 3 can be used to violate Bell's inequality. The Bell's inequality measurement consists essentially of four consecutive steps.

(1) Preparation step. During the preparation step, the two DQD's have to be held at the symmetry point, i.e., $\epsilon_{1}=\epsilon_{2}$ $=0$ and $\Delta_{1}=\Delta_{2}=\Delta$, where the Hamiltonian $H_{\mathrm{SP}}$ [see Eq. (20)] describes the two-qubit system. A measurement of the parity meter, i.e., the center QPC, is done. If the measurement is either $I_{\text {odd }}$ or $I_{\text {even }}$, then we know that either state $\left|\Psi_{B 1}\right\rangle$ or state $\left|\Psi_{B 2}\right\rangle$, respectively, has successfully been prepared.

(2) Decoupling step. Once we know that the system is in

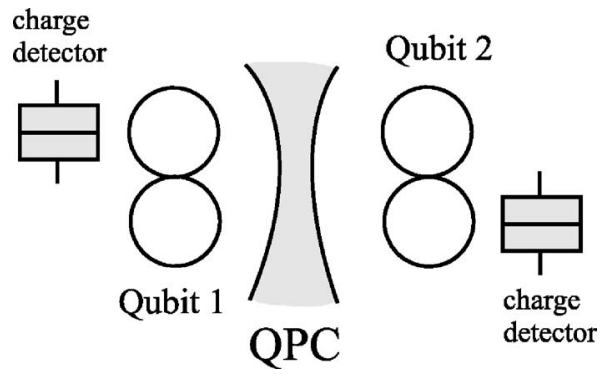

FIG. 3. Bell inequality setup. In order to be able to do a measurement of a violation of a Bell inequality, we use a QPC as a parity meter between two qubits to create a Bell state. The two outer detectors are then used to projectively measure charge in the $\sigma_{z}$ basis of each qubit.

one of the two Bell states $\left|\Psi_{B 1}\right\rangle$ or $\left|\Psi_{B 2}\right\rangle$, we would like to turn the coupling between the two qubits off. In our setup, the coupling between the two DQD's can be minimized by setting $V_{\text {bias }}=0$ and by pinching off the QPC.

(3) Single-qubit rotation step. In order to do a measurement of a violation of a Bell inequality in the Clauser-HorneShimony-Holt (CHSH) form, ${ }^{18}$ we have to rotate each qubit and afterward measure qubit-qubit correlators in different bases. The single-qubit rotation can be done by pulsing $\Delta_{1}(t)$ and $\Delta_{2}(t)$ independently of each other. Thus, during this step, we drive the system away from the symmetry point. Note that this is the only option to measure the two qubits in different bases, because typical measurement devices for charge qubits can only measure in the $\sigma_{z}$ basis of the qubit. Therefore, we have to rotate the state instead of the measurement device (which is the usual practice in Bell inequality measurements with photons).

(4) Measurement step. Immediately after the single-qubit rotation step, we should be able to do a strong (projective) measurement in the $\sigma_{z}$ basis of the qubit using high-fidelity single shot detectors. This can, for instance, be accomplished by single electron transistors as illustrated in Fig. 3. The time delay between the two projective measurements is analogous to the relative phase between the beam splitters in the original CHSH proposal.

An alternative to fast time-resolved projective measurements required for both the Bell inequality, and the CNOT gate of the next section, is making a series of many weak "kicked" quantum nondemolition measurements at a repetition rate commensurate with the Rabi period of the qubits. In this alternative, the qubits are not detuned from their symmetry point, and single-qubit rotations are accomplished by simply waiting. ${ }^{19}$

We have to repeat the four steps many times with the same time delay to obtain the correlation function

$$
C_{\mathrm{ab}}=\left\langle(\mathbf{a} \sigma)_{1} \otimes(\mathbf{b} \sigma)_{2}\right\rangle
$$

of the direct product of a "spin" measurement in qubit 1 along unit vector $\mathbf{a}$ and in qubit 2 along unit vector $\mathbf{b}$. Note that in our proposal the different angles $\mathbf{a}, \mathbf{a}^{\prime}, \mathbf{b}$, and $\mathbf{b}^{\prime}$ are realized by an appropriate application of the single-qubit rotation step. According to Bell, correlations are nonclassical if we violate the inequality 


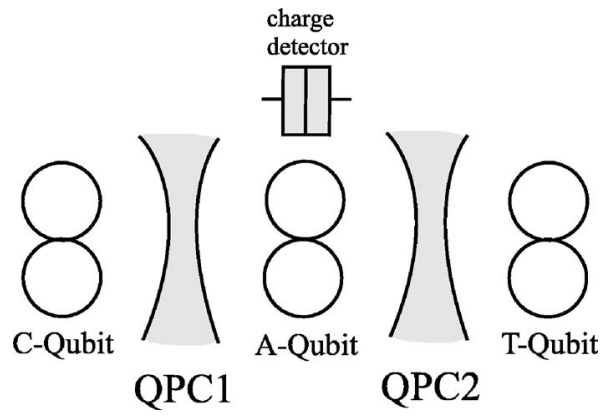

FIG. 4. CNOT gate setup. This setup contains three qubits and two parity meters, i.e., QPC's, in between them. The qubits are the control qubit ( $\mathrm{C}$ qubit), the ancilla qubit (A qubit), and the target qubit (T qubit). An additional projective measurement device is attached to the A qubit.

$$
\mathcal{B}=\left|C_{\mathbf{a b}}+C_{\mathbf{a}^{\prime} \mathbf{b}}+C_{\mathbf{a b}^{\prime}}-C_{\mathbf{a}^{\prime} \mathbf{b}^{\prime}}\right| \leq 2 .
$$

A simple way of analyzing the dephasing time in the system would be to choose different time delays after which the single-qubit rotation step sets in.

\section{B. CNOT gate}

The setup of a realization of a CNOT gate using charge parity meters is shown in Fig. 4 and follows the idea of Ref. 1. It consists of three different charge qubits and two parity meters. During the operation time of the CNOT gate $t_{\mathrm{op}}$ coherent evolution of the charge qubits should be negligible, which means that $\Delta t_{\mathrm{op}} \ll 1$. Furthermore, $t_{\mathrm{op}}$ has to be smaller than the typical dephasing time $T_{2}$ of the qubits. Note that in superconducting charge qubits, a CNOT gate operation has already been demonstrated experimentally ${ }^{20}$ and interesting proposals for the implementation of different kinds of two-qubit gates exist. ${ }^{21}$

The Hamiltonian of the CNOT gate may be written as

$$
H_{\mathrm{CNOT}}=H_{\mathrm{QD}}+H_{\mathrm{QPC}}+H_{\mathrm{int}}
$$

with

$$
\begin{gathered}
H_{\mathrm{QD}}=-\frac{1}{2} \sum_{\alpha=1,2,3}\left(\epsilon_{\alpha} \sigma_{z}^{(\alpha)}+\Delta_{\alpha} \sigma_{x}^{(\alpha)}\right), \\
H_{\mathrm{QPC}}=H_{\mathrm{QPC}, 1}+H_{\mathrm{QPC}, 2}, \\
H_{\mathrm{int}}=\frac{\Delta \hat{E}_{1}}{2} \sigma_{z}^{(1)} \sigma_{z}^{(2)}+\frac{\Delta \hat{E}_{2}}{2} \sigma_{z}^{(2)} \sigma_{z}^{(3)} .
\end{gathered}
$$

The energies $\epsilon_{\alpha}$ and $\Delta_{\alpha}(\alpha=1,2,3)$ can (in principle) be controlled at any time by changing the gate voltages that determine the single-particle levels and the tunnel couplings in each DQD system, respectively.

We now assume that the control qubit and the target qubit are in a given state. It is important for the scheme to work that the ancilla qubit is prepared in the state $\left|\Psi_{\mathrm{AQ}}\right\rangle=(|\uparrow\rangle$ $+|\downarrow\rangle) / \sqrt{2}$. It should be rather easy to prepare the desired state of the ancilla qubit because it is the ground state of the DQD system when the tunnel coupling is finite. ${ }^{22}$ Under the constraint $\Delta t_{\mathrm{op}} \ll 1$, mentioned above, the outcome of the parity measurement can just take two values, corresponding to $I_{\text {odd }}$ or $I_{\text {even }}$ of Sec. III. Then, a measurement of $I_{\text {odd }}$ shows that the two qubits involved in that measurement exist in the subspace spanned by the states $|\uparrow \downarrow\rangle$ and $|\downarrow \uparrow\rangle$, whereas a measurement of $I_{\text {even }}$ indicates that the two qubits involved in that measurement exist in the subspace spanned by the states $|\uparrow \uparrow\rangle$ and $|\downarrow \downarrow\rangle$.

Apart from the measurements of the two parity meters, the only other ingredients needed to build a deterministic CNOT gate are single-qubit Hadamard gates $\mathcal{H}=\left(\sigma_{x}+\sigma_{z}\right) / \sqrt{2}$. A Hadamard gate acting on qubit $\alpha$ can be realized by tuning the corresponding single-qubit Hamiltonian

$$
\mathcal{H}_{\alpha}=-\frac{1}{2}\left(\epsilon_{\alpha} \sigma_{z}^{(\alpha)}+\Delta_{\alpha} \sigma_{x}^{(\alpha)}\right)
$$

to the special symmetry point $\epsilon_{\alpha}=\Delta_{\alpha}$ and letting it act for a time $t_{H}=\pi / \sqrt{2} \Delta$. For this single-qubit rotation, we need to raise $\Delta$ and $\epsilon$ temporarily.

The operation scheme of the CNOT gate goes as follows.

(1) Preparation of the ancilla qubit. This can be either done by acting with $\mathcal{H}_{2}$ on state $|0\rangle$ of qubit 2 or by ground state preparation of a tunnel-coupled double dot.

(2) Parity measurement with QPC 1. The outcome of that measurement $p_{1}$ has to be stored. $p_{1}=0$ corresponds to the odd parity class, whereas $p_{1}=1$ corresponds to the even parity class. The same holds, of course, for $p_{2}$, i.e., the outcome of the parity measurement 2 below. Afterward, we need to decouple the control and the ancilla qubit.

(3) Hadamard step 1. Acting with $\mathcal{H}_{2}$ on the ancilla qubit and with $\mathcal{H}_{3}$ on the target qubit, which means in practice to wait for an appropriate time $t_{H}$ after tuning the single-qubit Hamiltonians.

(4) Parity measurement with QPC 2. The outcome of that measurement $p_{2}$ has to be stored. Afterward, we need to decouple the ancilla and the target qubit.

(5) Hadamard step 2. Once more, acting with $\mathcal{H}_{2}$ on the ancilla qubit and with $\mathcal{H}_{3}$ on the target qubit.

(6) Measurement of ancilla qubit. This measurement has to be done in a projective way.

(7) Postprocessing step. Depending on the outcome of the measured state of the ancilla qubit as well as $p_{1}$ and $p_{2}$, we finally have to apply single-qubit operations to the control and the target qubit, which we call $\sigma_{c}$ and $\sigma_{t}$. For the control qubit, $\sigma_{c}=\sigma_{z}$ if $p_{2}=0$, while no postprocessing of the control qubit is needed if $p_{2}=1$. For the target qubit, $\sigma_{t}=\sigma_{x}$ if the ancilla qubit is down and $p_{1}=1$, or if the ancilla qubit is up and $p_{1}=0$. Otherwise, no postprocessing of the target qubit is needed. Applying a conditional operation of $\sigma_{x}$ or $\sigma_{z}$, this means in practice making $\Delta_{\alpha} \gg \epsilon_{\alpha}$ for the former case or vice versa for the latter one.

As demonstrated in detail in Ref. 23, the different steps described above allow for a CNOT operation on the control and the target qubits.

\section{CONCLUSIONS}

We have proposed a realization of a charge parity meter, i.e., a device that can distinguish between the subspaces of 
two parity classes of quantum states but cannot distinguish between different states in each parity class. If the states are two-qubit states (in our case, the states that characterize two charge qubits) a parity meter based on a QPC placed in a proper way between the two qubits acts as an entangler just by a current measurement. Such a device is a specific realization of a mesoscopic quadratic quantum measurement. ${ }^{4}$

Furthermore, we have demonstrated that the charge parity meter supplemented by two single-qubit charge detectors can be used to do a measurement of a violation of Bell's inequality in the solid state. To accomplish this, we have exploited the idea of using single-qubit rotations instead of a rotation of the measurement device in order to be able to measure $\mathrm{CHSH}$ correlators in four different bases.

Finally, a CNOT gate operation has been described using two parity meters and three qubits. Thereby, one of the three qubits just acts as an ancilla qubit to enable a deterministic gate, whereas the other two qubits are the standard control and target qubits that are manipulated by the CNOT operation.

\section{ACKNOWLEDGMENTS}

Interesting discussions with N. Gisin, F. H. L. Koppens, L. P. Kouwenhoven, and L. M. K. Vandersypen are gratefully acknowledged. A.N.J. thanks the Kavli Institute of Nanoscience at the TU Delft for kind hospitality. Financial support was provided by the Dutch Science Foundation NWO/FOM, the Swiss NSF, and MaNEP. We acknowledge support by the EC's Marie Curie Research Training Network under Contract No. MRTN-CT-2003-504574, Fundamentals of Nanoelectronics. The work of A.N.J. has been supported by AFRL Grant No. F30602-01-1-0594, AFOSR Grant No. FA955004-1-0206, and TITF Grant No. 2001-055.
${ }^{1}$ C. W. J. Beenakker, D. P. DiVincenzo, C. Emary, and M. Kindermann, Phys. Rev. Lett. 93, 020501 (2004).

${ }^{2}$ H.-A. Engel and D. Loss, Science 309, 586 (2005).

${ }^{3}$ For a perspective on these developments, see J. Carlos Egues, Science 309, 565 (2005).

${ }^{4}$ W. Mao, D. V. Averin, R. Ruskov, and A. N. Korotkov, Phys. Rev. Lett. 93, 056803 (2004).

${ }^{5}$ R. Ruskov and A. N. Korotkov, Phys. Rev. B 67, 241305(R) (2003).

${ }^{6}$ T. Hayashi, T. Fujisawa, H. D. Cheong, Y. H. Jeong, and Y. Hirayama, Phys. Rev. Lett. 91, 226804 (2003).

${ }^{7}$ S. A. Gurvitz, Phys. Rev. B 56, 15215 (1997); A. N. Korotkov, ibid. 63, 085312 (2001); A. N. Korotkov and D. V. Averin, ibid. 64, 165310 (2001); S. Pilgram and M. Büttiker, Phys. Rev. Lett. 89, 200401 (2002); A. A. Clerk, S. M. Girvin, and A. D. Stone, Phys. Rev. B 67, 165324 (2003); D. V. Averin and E. V. Sukhorukov, Phys. Rev. Lett. 95, 126803 (2005); A. N. Jordan and M. Büttiker, ibid. 95, 220401 (2005); A. A. Clerk, Phys. Rev. Lett. 96, 056801 (2006).

${ }^{8}$ J. R. Petta, A. C. Johnson, C. M. Marcus, M. P. Hanson, and A. C. Gossard, Phys. Rev. Lett. 93, 186802 (2004); J. M. Elzerman, R. Hanson, L. H. Willems van Beveren, B. Witkamp, L. M. K. Vandersypen, and L. P. Kouwenhoven, Nature (London) 430, 431 (2004); J. R. Petta, A. C. Johnson, J. M. Taylor, E. A. Laird, A. Yacoby, M. D. Lukin, C. M. Marcus, M. P. Hanson, and A. C. Gossard, Science 309, 2180 (2005); F. H. L. Koppens, J. A. Folk, J. M. Elzerman, R. Hanson, L. H. Willems van Beveren, I. T. Vink, H. P. Tranitz, W. Wegscheider, L. P. Kouwenhoven, and L. M. K. Vandersypen, ibid. 309, 1346 (2005).

${ }^{9}$ N. Lambert, R. Aguado, and T. Brandes, cond-mat/0602063 (unpublished).

${ }^{10}$ S. Vorojtsov, E. R. Mucciolo, and H. U. Baranger, Phys. Rev. B 71, 205322 (2005); Z.-J. Wu, K.-D. Zhu, X.-Z. Yuan, Y.-W. Jiang, and H. Zheng, Phys. Rev. B 71, 205323 (2005).
${ }^{11}$ T. Itakura and Y. Tokura, Phys. Rev. B 67, 195320 (2003).

${ }^{12}$ N. Mason, M. J. Biercuk, and C. M. Marcus, Science 303, 655 (2004); S. Sapmaz, C. Meyer, P. Beliczynski, P. Jarillo-Herrero, and L. P. Kouwenhoven, cond-mat/0602424 (unpublished).

${ }^{13}$ J. Gorman, E. G. Eminoglu, D. G. Hasko, and D. A. Williams, Phys. Rev. Lett. 95, 090502 (2005).

${ }^{14}$ R. Ruskov, A. N. Korotkov, and A. Mizel, Phys. Rev. B 73, 085317 (2006).

${ }^{15}$ M. Büttiker, Phys. Rev. B 41, R7906 (1990).

${ }^{16}$ T. Christen and M. Büttiker, Phys. Rev. Lett. 77, 143 (1996).

${ }^{17}$ L. M. K. Vandersypen, J. M. Elzerman, R. N. Schouten, L. H. Willems van Beveren, R. Hanson, and L. P. Kouwenhoven, Appl. Phys. Lett. 85, 4394 (2004).

${ }^{18}$ J. F. Clauser, M. A. Horne, A. Shimony, and R. A. Holt, Phys. Rev. Lett. 23, 880 (1969).

${ }^{19}$ A. N. Jordan and M. Büttiker, Phys. Rev. B 71, 125333 (2005); A. N. Jordan, A. N. Korotkov, and M. Büttiker, cond-mat/ 0510782 (unpublished).

${ }^{20}$ T. Yamamoto, Yu. A. Pashkin, O. Astafiev, Y. Nakamura, and J. S. Tsai, Nature (London) 425, 941 (2003).

${ }^{21}$ L. F. Wei, Yu-xi Liu, M. J. Storcz, and F. Nori, Phys. Rev. A 73, 052307 (2006).

${ }^{22}$ W. G. van der Wiel, S. De Franceschi, J. M. Elzerman, T. Fujisawa, S. Tarucha, and L. P. Kouwenhoven, Rev. Mod. Phys. 75, 1 (2003).

${ }^{23}$ C. W. J. Beenakker, D. P. DiVincenzo, C. Emary, and M. Kindermann, quant-ph/0401066, Appendix A.

${ }^{24}$ We have derived the Hamiltonian (3) in the scattering picture of transport through the detector. On the level of observables such as the average current and the noise, the scattering picture of transport and the tunneling picture of transport are equivalent in the tunneling regime. The validity range of the scattering picture, however, also extends to the regime of an open QPC. 